\title{
Detection and Structural Characterization of Glutathione-Trapped Reactive Metabolites Using Liquid Chromatography-High Resolution Mass Spectrometry and Mass Defect Filtering
}

\author{
Mingshe Zhu*, Li Ma, Haiying Zhang and W. Griffith Humphreys \\ Biotransformation, Pharmaceutical Candidate Optimization, Bristol-Myers \\ Squibb Research and Development, Princeton, NJ 08543 \\ Supplement information \\ Table of content
}

Figure 1. Accurate mass full scan MS spectra of GSH adducts and stable oxidative metabolites of clozapine formed in an HLM incubation supplemented with GSH.

Figure 2. MDF processed profiles of GSH adducts of $p$-cresol, acetaminophen and diclofenac formed in HLM incubations (10 $\mu \mathrm{M}$ substrate concentration) supplemented with GSH.

Figure 3. MDF analysis of GSH-trapped reactive metabolites of carbamazepine (10 $\mu \mathrm{M})$ in an HLM incubation supplemented with GSH.

Figure 4. MDF processed full scan MS spectra of GSH adducts of carbamazepine. 
Figure 1. Accurate mass full scan MS spectra of GSH adducts and stable oxidative metabolites of clozapine formed in an HLM incubation supplemented with GSH. (A) Clozapine- $N$-oxide. (B) Desmethylclozapine. (C) OM3. (D) OM2. The accurate mass full scan MS spectral data were acquired by LC/FTMS.
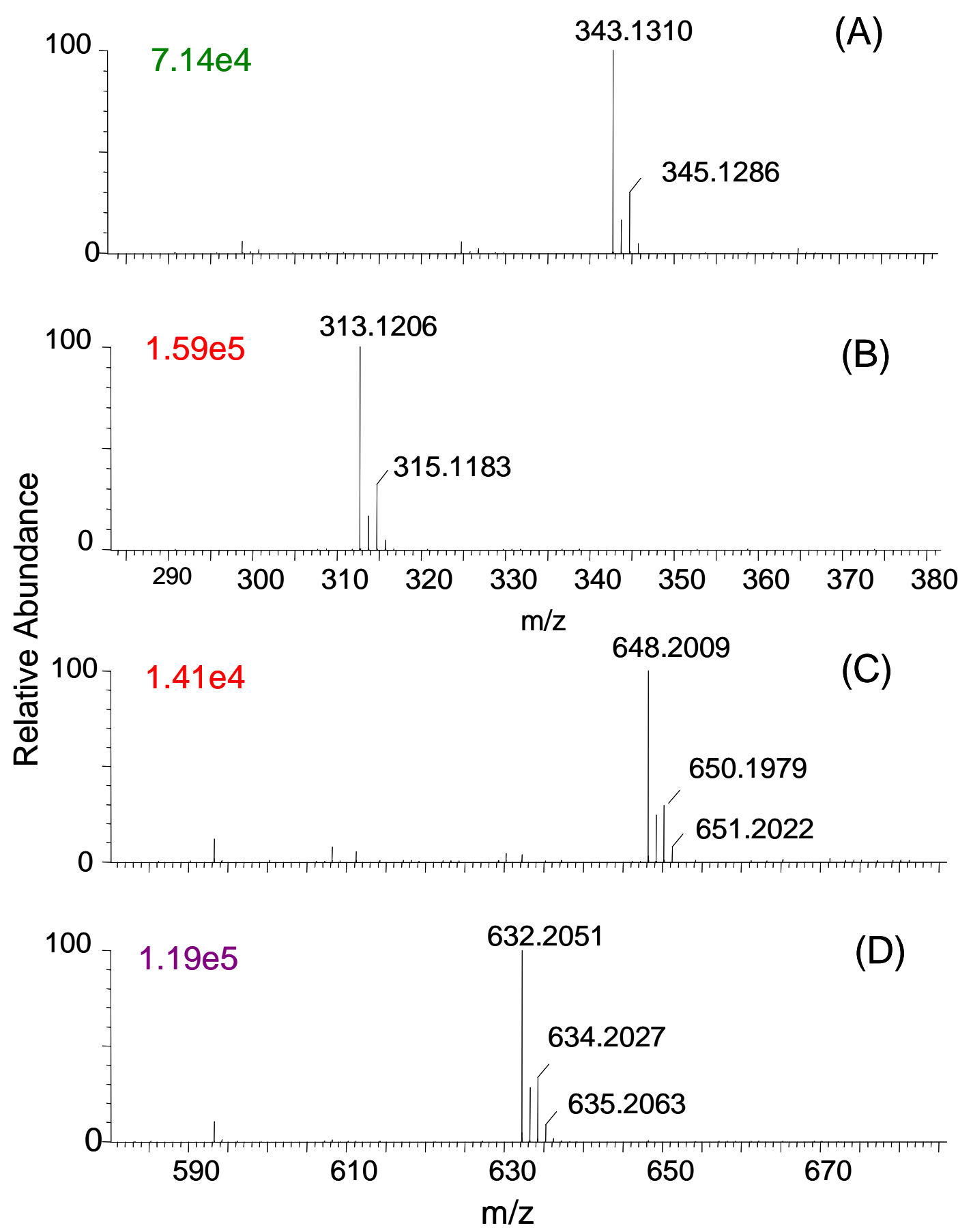
Figure 2. MDF processed profiles of GSH adducts of $p$-cresol, acetaminophen and diclofenac formed in HLM incubations (10 $\mu \mathrm{M}$ substrate concentration) supplemented with GSH. (A) p-Cresol. (B) Acetaminophen. (C) Diclofenac.
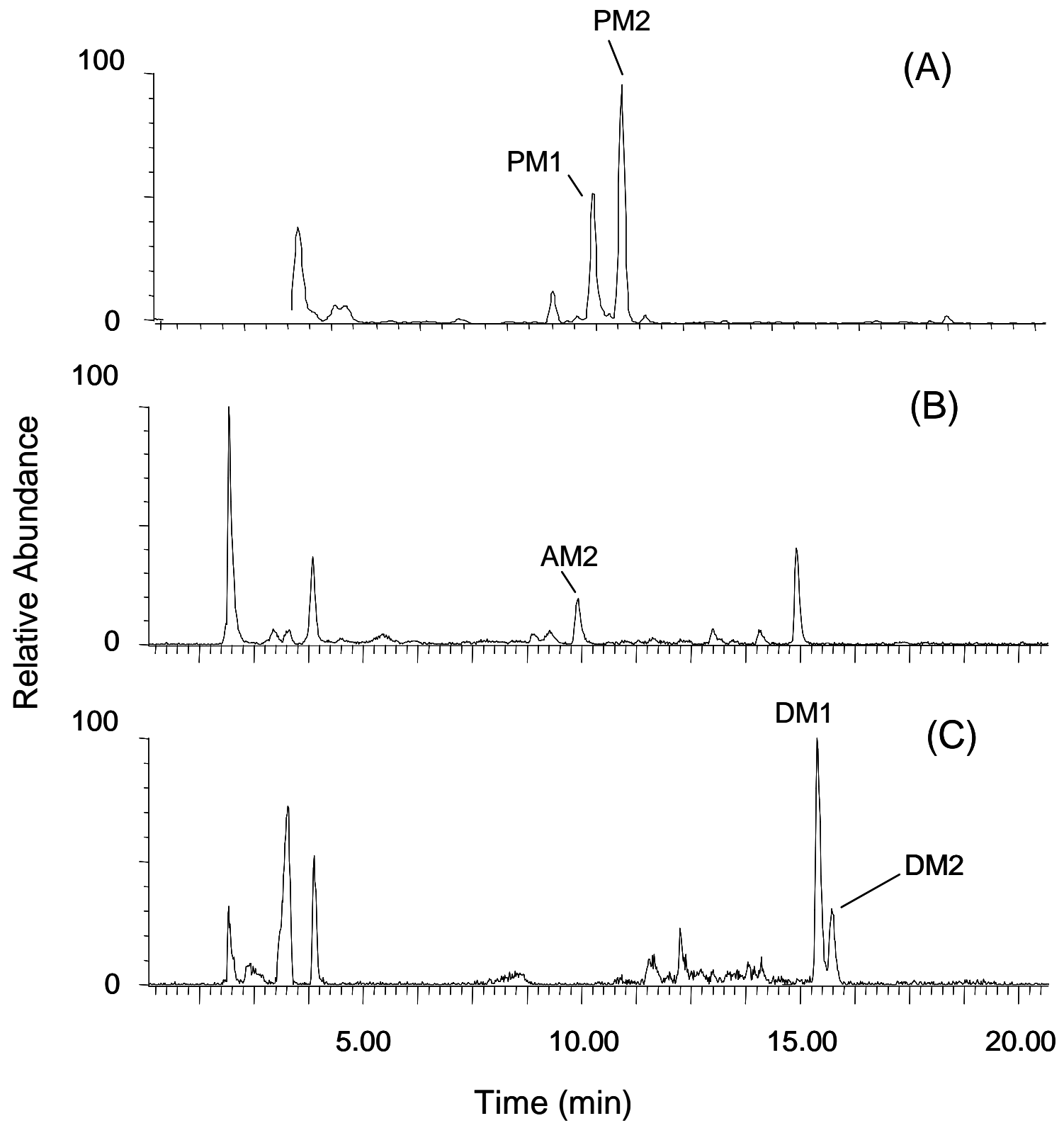
Figure 3. MDF analysis of GSH-trapped reactive metabolites of carbamazepine (10 $\mu \mathrm{M})$ in an HLM incubation supplemented with GSH. (A) TIC of unprocessed accurate mass full scan LC/MS data. (B) MDF-processed ion chromatogram obtained using the drugGSH adduct filter template. (C) EIC of the MDF-processed LC/MS data for $\mathrm{m} / \mathrm{z} 576$. (D) EIC of the MDF-processed LC/MS data for $\mathrm{m} / \mathrm{z} 560$. (E) EIC of the MDF-processed LC/MS data for $m / z$ 558. The accurate mass LC/MS data were acquired by LC/FTMS.

(A)

Scan ES+

TIC

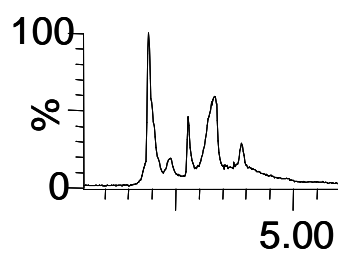

$4.71 \mathrm{e} 6$
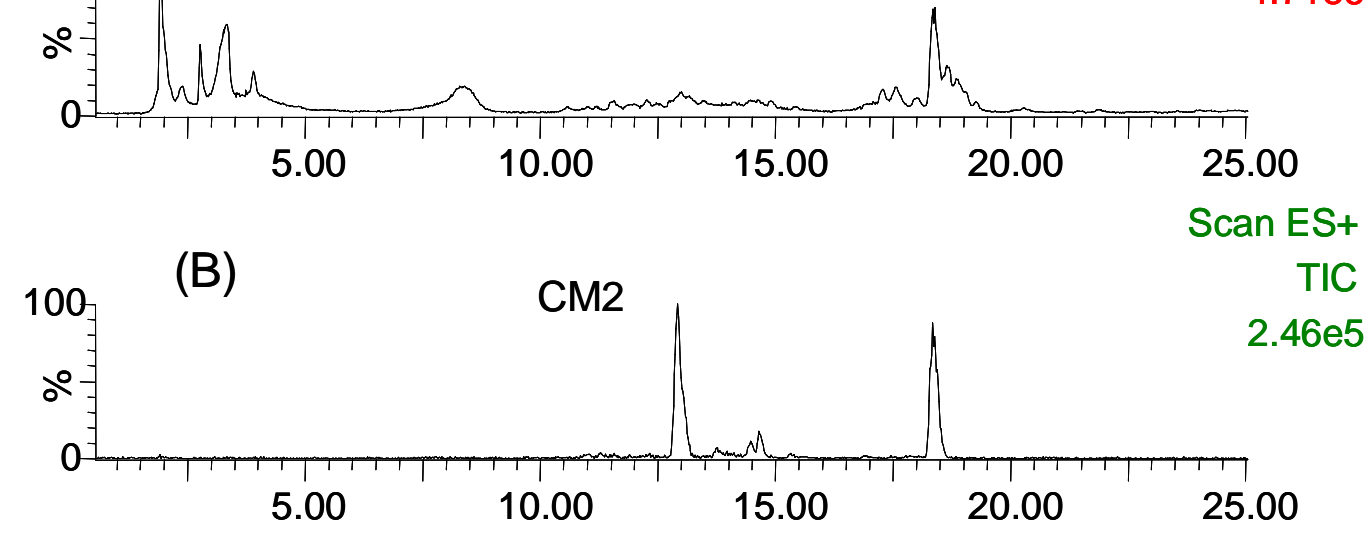

10.00

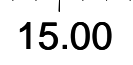

Scan ES+
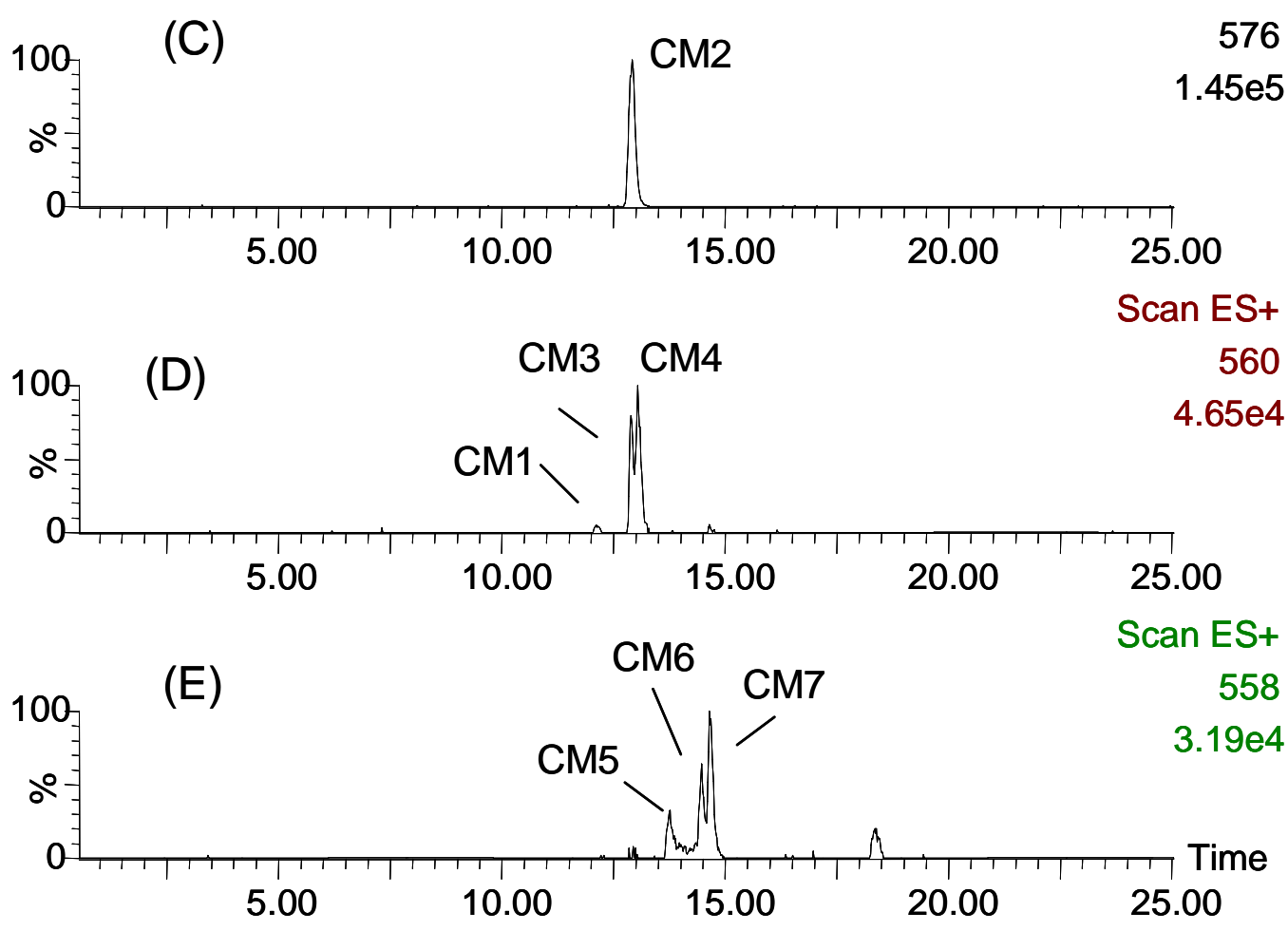

$\mathrm{m} / \mathrm{z}$ 
Figure 4. MDF processed full scan MS spectra of GSH adducts of carbamazepine. (A) CM2. (B) CM3. (C) CM7. The accurate mass LC/MS data were acquired by LC/FTMS.

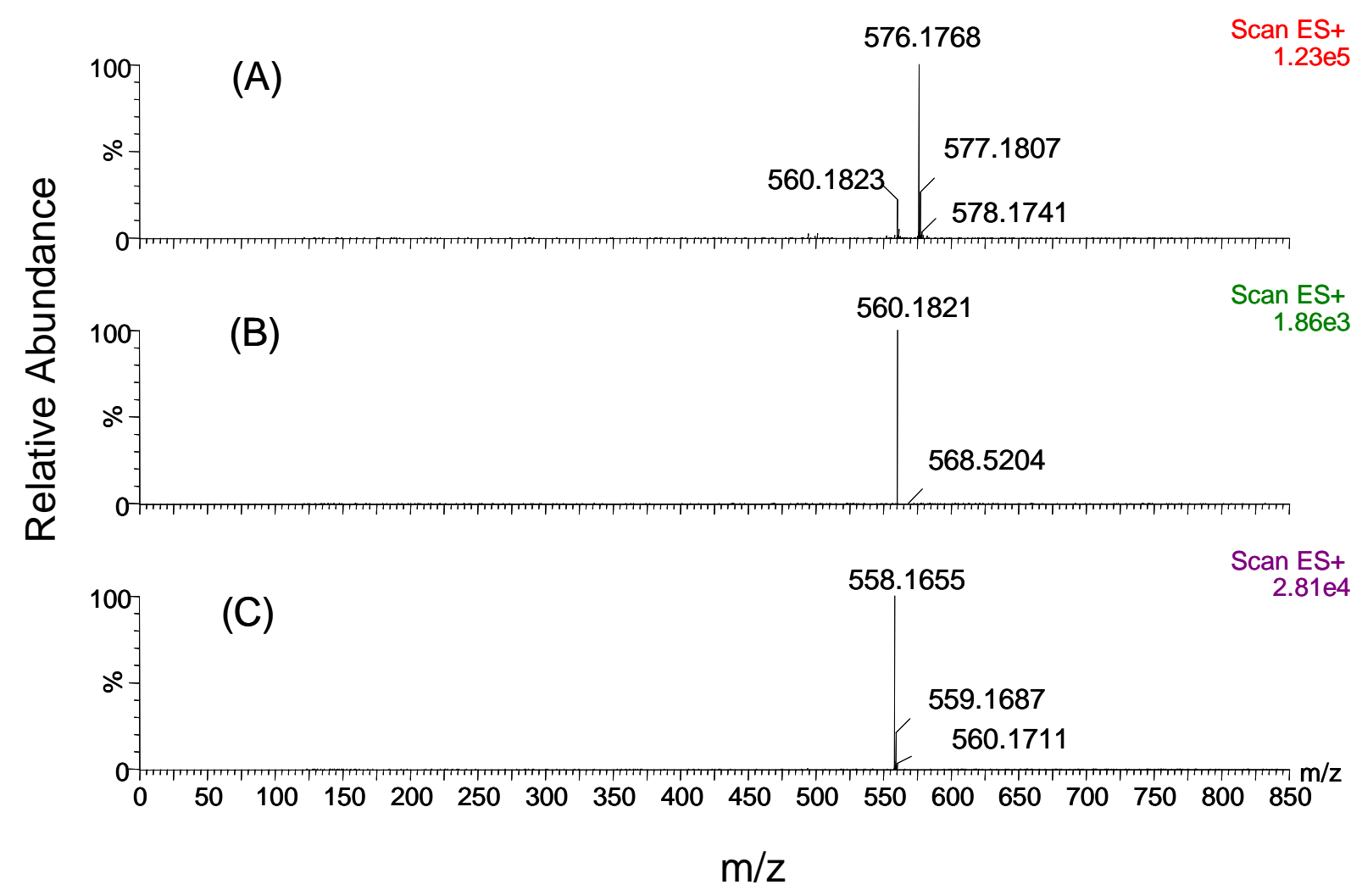

